\title{
IGF and mTOR pathway expression and in vitro effects of linsitinib and mTOR inhibitors in adrenocortical cancer
}

\author{
Maria Cristina De Martino $\mathbb{D}^{1,2} \cdot$ Peter M. van Koetsveld ${ }^{1} \cdot$ Richard A. Feelders $^{1} \cdot$ Wouter W. de Herder $^{1}$. \\ Fadime Dogan ${ }^{1}$ - Joseph A. M. J. L. Janssen ${ }^{1} \cdot$ Davine Hofste op Bruinink ${ }^{1} \cdot$ Claudia Pivonello $^{2}$ - A. Marlijn Waaijers ${ }^{1}$. \\ Annamaria $\mathrm{Colao}^{2} \cdot{\text { Ronald R. de } \mathrm{Krijger}^{3} \cdot \text { Rosario Pivonello }^{2} \cdot \text { Leo J. Hofland }}^{1}$
}

Received: 8 October 2018 / Accepted: 12 February 2019 / Published online: 5 March 2019

(c) The Author(s) 2019

\begin{abstract}
Purpose The IGF and mTOR-pathways are considered as potential targets for therapy in patients with adrenocortical carcinoma (ACC). This study aims to describe the IGF pathway in ACC and to explore the response to the combined treatment with the IGF1R/IR inhibitor linsitinib, and mTOR inhibitors (sirolimus and everolimus) in in vitro models of ACC. Methods The protein expression level of IGF2, IGF1R and IGF2R was evaluated by immunohistochemistry in 17 human ACCs and the mRNA expression level of IGF1,IGF2,IGFIR, IR isoforms $A$ and B,IGF2R, IGF-Binding-Proteins $[I G F B P]-1,2,3$ and 6 was evaluated by RT-qPCR in 12 samples. In H295R and HAC15 ACC cell lines the combined effects of linsitinib and sirolimus or everolimus on cell survival were evaluated.

Results A high protein expression of IGF2, IGF1R and IGF2R was observed in 82, 65 and 100\% of samples, respectively. A high relative expression of IGF2 mRNA was found in the majority of samples. The mRNA levels of the IRA were higher than that of $I R B$ and $I G F I R$ in the majority of samples (75\%). Linsitinib inhibits cell growth in the H295R and HAC15 cell lines and, combined with sirolimus or everolimus, linsitinib showed a significant additive effect.

Conclusions In addition to IGF2 and IGF1R, ACC express IGF2R, IRA and several IGFBPs, suggesting that the interplay between the different components of the IGF pathway in ACC could be more complex than previously considered. The addition of mTOR inhibitors to linsitinib may have stronger antiproliferative effects than linsitinib alone.
\end{abstract}

Keywords: Adrenal $\cdot$ Linsitinib $\cdot$ IGF $\cdot$ Insulin receptor $\cdot$ Adrenocortical cancer

\section{Introduction}

Malignant tumors of the adrenal cortex, defined as adrenocortical carcinoma (ACC), are rare but aggressive cancers for which new treatment options are required [1-4].

Supplementary information The online version of this article (https:// doi.org/10.1007/s12020-019-01869-1) contains supplementary material, which is available to authorized users.

Leo J. Hofland

1.hofland@erasmusmc.nl

1 Department of Internal Medicine, Division Endocrinology, Erasmus Medical Center, Rotterdam, The Netherlands

2 Dipartimento di Medicina Clinica e Chirurgia, Università Federico II, Naples, Italy

3 Departments of Pathology, Erasmus Medical Center, Rotterdam, and University Medical Center Utrecht, Utrecht, The Netherlands
Although most ACCs are sporadic, ACCs rarely develop in the context of certain genetic syndromes such as the Beckwith-Wiedemann syndrome (BWS), Li-Fraumeni and familial colorectal polyposis. The study of these syndromes has supported the potential role of some molecular pathways in ACC pathogenesis [5]. Particularly, the BWS is a genetic syndrome associated with childhood ACC, other childhood tumors and a somatic overgrowth syndrome in which deregulation of imprinted genes on chromosomal locus $11 \mathrm{p} 15$ leads to biallelic expression of $I G F 2[5,6]$. Although the estimated prevalence of BWS in patients with ACC is very low and restricted to the childhood [5, 7], IGF2 has been reported to be over-expressed in about $70-90 \%$ of sporadic ACCs as compared to normal adrenals or benign adrenocortical tumors [8-17]. Therefore, the insulin-like growth factor (IGF) system is considered as a promising target for new medical treatment options in ACC $[11,14,18]$. The IGF system participates in the regulation of growth, lifespan and metabolism and includes circulating ligands, exerting their 
effects as endocrine and/or paracrine factors [insulin, IGF1 and IGF2 (IGFs)]; binding proteins (IGFBP1-6 that modulate the bioavailability of IGFs) and multiple receptors [19]. Among the receptors, the IGF1 receptor (IGF1R) and the insulin receptor isoforms $\mathrm{A}$ and $\mathrm{B}$ (IRA and IRB) are tyrosine-kinase receptors. The mannose 6-phosphate/insulin-like growth factor 2 receptor (IGF2R) is a scavenger receptor involved in the internalization and degradation of IGF2. In adult humans, insulin predominantly exerts metabolic effects through the activation of IRB, whereas IGFs, particularly IGF1, mainly exerts growth-stimulating effects through the activation of IGF1R receptors. IRA is predominantly expressed during fetal development when it is an important mediator of pro-growth effects of insulin and IGFs. IRA and its expression in malignant tumor tissue has been suggested to be involved in cancer development [19, 20]. Currently, the efficacy of several IGF1R and IGF1R/IR inhibitors is evaluated in clinical trials, alone or in combination with other agents for the treatment of several malignant disorders [21-23]. mTOR is a protein kinase of the phosphatidylinositol 3-kinase (PI3K)/Akt/mTOR signaling pathway and plays a pivotal role in cell growth, metabolism and proliferation, by mediating the effects of various growth factors, including the IGFs [24]. The mTOR pathway is considered a target for antineoplastic therapy in several malignancies and it has recently been proposed as a target for ACC treatment [25-28].

This study aims at describing the IGF pathway in ACC and to explore the in vitro response to the combined treatment with a dual IGF1R/IR inhibitor (linsitinib) and the mTOR inhibitor (sirolimus) in an in vitro model of ACC using ACC cell lines.

\section{Materials and methods}

\section{Subjects}

Seventeen ACCs and 6 normal adrenal tissue samples (NA) samples were used for this study. Fresh tissue was snap frozen within 60 minutes after surgical removal. NA samples were collected for in vitro studies from adrenalectomy (NA) due to renal cell carcinoma. This study was approved by the Medical Ethics Committee of the Erasmus MC and all patients gave written informed consent.

The following clinical parameters were recorded in all patients: date of diagnosis, age, gender, ENSAT stage [29], Weiss score (assessed by an expert pathologist in adrenal disease [RRdK]) [30], mitotic count (as defined by the presence number of mitoses equal or higher than 5 in 50 high-power fields), hormonal status and type of hormonal secretion (cortisol and/or androgens and/or estrogens and/or mineralocorticoids) [31].

\section{Total RNA isolation and quantitative RT-PCR (RT-qPCR)}

From snap frozen adrenal tissues (available for 12 ACCs cases and 6 NA cases), total RNA was isolated using a commercially available kit (High Pure RNA Tissue kit; Roche, Almere, The Netherlands).

Total RNA from the human ACC cell line NCI-H295R (H295R) was used as a positive control.

The cDNA synthesis from total RNA and quantitative PCR were performed as previously described [25]. mRNA expression of IGF1, IGF2, IGF1R, IRA, IRB, IGF2R, IGFBP 1, 2, 3 and 6 and of the housekeeping gene hypoxanthine phosphoribosyltransferase (HPRT) was evaluated by RT-qPCR in human ACC tissue samples, depending on the availability of frozen tissues.

The primers and probes were purchased from SigmaAldrich (Zwijndrecht, The Netherlands) and are reported in the Supplemental table 1. Samples were normalized to the expression of HPRT. PCR efficiencies (E) were calculated for the primer-probe combinations used [32]. The relative expression of genes was calculated using the comparative threshold method, $2^{-\Delta \mathrm{Ct}}$ [33], after efficiency correction [34] of target and reference gene transcripts (HPRT).

\section{Immunohistochemistry (IHC)}

The expression of IGF2, IGF1R and IGF2R in adrenal samples was evaluated. Paraffin embedded tissue specimens were cut in $5 \mu \mathrm{m}$ sections, deparaffinized and dehydrated. Antigen-retrieval was performed by microwave treatment in Tris-EDTA Buffer ( $\mathrm{pH}$ 9.0). The slides were cooled for $1 \mathrm{~h}$ at $+4{ }^{\circ} \mathrm{C}$ and incubated for $1 \mathrm{~h}$ at room temperature (RT) with the primary monoclonal antibodies and incubated overnight at $+4{ }^{\circ} \mathrm{C}$ with the primary polyclonal antibodies. The primary monoclonal antibodie to detect IGF1R was purchased from Novus Biologicals (NB110-87052; dilution: 1:500) and the primary polyclonal antibodies to detect IGF2 and IGF2R were purchased from R\&D Systems (AF-292-NA; dilution: 1:500) and Santa Cruz Biotech (SC-25462; dilution: 1:50) respectively. The slides were washed and incubated for $30 \mathrm{~min}$ at RT with secondary antibodies (Poly-AP-Goat anti-Mouse/Rabbit IgG PowerVision + ; ImmunoVision Technologies) at the concentration provided by the manufacturer. After washing, staining was visualized by a $30 \mathrm{~min}$ incubation in new fuchsin solution. Only IGF1R staining was performed and visualized with a Dako Detection System, following a different protocol previously described [25]. All slides were counterstained with hematoxylin and coverslipped. Positive controls included cases of adrenocortical cancer and normal human pancreas with previously proven positivity at IHC for the protein evaluated. Negative controls included omission of the primary antibody and the incubation with secondary antibodies. 
The staining was evaluated independently by two investigators and any discrepancy was resolved by a consensus review. The results were interpreted in a semiquantitative manner by using an intensity-proportion scoring system previously described [35]. The score was calculated by the sum of the intensity score and the proportion of the stained cells; this provided a score between 0 and 6 . The proportion score was as follows: $0=$ no positivity (or less than $10 \%$ ); $+1=$ less than $1 / 3$ tumor cell positivity $+2=1 / 3$ to $2 / 3$ tumor cell positivity; and $+3=$ more than $2 / 3$ tumor cell positivity. The intensity score was as follows: $+1=$ weak staining $+2=$ intermediate staining; $+3=$ strong staining. The score 0 was regarded as negative; $2-3$ as low; $4-5$ as intermediate and 6 as high. Finally adrenocortical tumors were dichotomously grouped as having intermediate to high expression of the evaluated protein and phospho-proteins (IHC score equal-higher than 4) or not (IHC score lower than 4).

\section{Drugs and reagents}

The dual IGF1R/IR inhibitor linsitinib and the mTOR inhibitors sirolimus and everolimus were purchased from LC Laboratories (Inc. Woburn, MA, USA) and prepared as a $10^{-3} \mathrm{M}$ stock solution in dimethylsulfoxide (DMSO). Compounds were stored at $-20{ }^{\circ} \mathrm{C}$ and further diluted in $40 \%$ DMSO before the use. Final DMSO concentration, also added as vehicle to controls, was $0.4 \%$.

\section{Cell lines and culture conditions}

The human ACC cell lines H295R and HAC15 were obtained from the American Type Culture Collection (Manassas, VA) and from Dr. W. Rainey (as gift), respectively. Short Tandem Repeat (STR) profiling using a Powerplex Kit (Promega) of H295R gave results consistent with those described in the ATCC database, thus confirming the H295R cell line identity. STR profiling of HAC15 showed that HAC15 has a genetic profile identical to $\mathrm{H} 295 \mathrm{R}$, which is consistent with a previous report by Rainey et al. that HAC15 is a clone of H295R [36]. The cells were cultured as previously described in detail [25] and utilized up to the $15^{\text {th }}$ passage.

\section{Measurement of total DNA content assay}

Measurement of total DNA content per well was used to determine the effects of the compounds on cell proliferation. Cells were plated in $1 \mathrm{ml}$ of medium in 24-well plates at the density required to obtain a $65-70 \%$ cell confluence in the control groups at the end of the experiment. The experiments were performed using medium containing high
(5\% FCS) or low serum (1\% FCS). Twenty-four hours later compounds were added to wells in quadruplicate, medium was refreshed at day 3 and fresh compounds were added again. After 3 or 6 days of treatment with the selected compounds, cells were harvested for DNA measurement, as a measure of cell number. All controls were vehicle treated. Measurement of total DNA content was previously described in detail [37].

\section{Apoptosis assay}

Apoptosis has been studied using two methods: "DNA fragmentation assay" and "Muse ${ }^{\mathrm{TM}}$ Annexin V \& Dead Cell Kit".

\section{DNA fragmentation assay}

The cells were plated in 24-well plates and treated as above described for the cell proliferation assay. After $24 \mathrm{~h}$ compounds or vehicle were added and after 3 days of incubation, DNA fragmentation was determined using a commercially available ELISA kit (Roche Diagnostic $\mathrm{GmbH}$, Penzberg, Germany). The standard protocol supplied by the manufacturer was used. The same plates were also analyzed for the measurement of total DNA content. The amount of DNA-fragmentation (apoptosis) was corrected for the total DNA content in each well.

\section{Muse $^{\mathrm{TM}}$ Annexin V \& Dead Cell Kit (Millipore, Germany)}

Cells were plated in 12-well plates at the density necessary to obtain a $65-70 \%$ cell confluence in the control groups at the end of the experiment. Twenty-four hours later, sirolimus was added to wells in duplicate. Control groups were vehicle-treated. After seventy-two hours of treatment, cells were harvested by gentle trypsinization and processed for staining according to the protocol provided by the supplier of the assay. The experiments were repeated twice.

\section{Cell cycle assay}

The effects of compounds on cell cycle progression were evaluated using the "Muse ${ }^{\mathrm{TM}}$ Cell Cycle Assay" (Millipore, Germany). Cells were plated in 12-well plates at the density necessary to obtain a $65-70 \%$ cell confluence in the control groups at the end of the experiment. Twenty-four hours later sirolimus was added to wells in duplicate. Control groups were vehicle-treated. After seventy-two hours of treatment, cells were harvested by gentle trypsinization and processed for fixation and staining according to the protocol provided by the supplier of the assay. The experiments were repeated twice. 


\section{Statistical analysis}

All the experiments were carried out at least three times, with the exception of the apoptosis assays and cell cycle assay that were performed twice. The repeated experiments gave comparable results. For the statistical analysis, statistical software SPSS (SPSS 15.0; SPSS Inc., Chicago, IL) and GraphPad Prism 5.0 (GraphPhad Software, San Diego, CA) were used. The Spearman's rank coefficient (rho) was used to test correlation.

We used non-parametric tests to evaluate the differences among groups (Mann-Whitney and Kruskall-Wallis). The comparative statistical evaluations among treatment groups were performed by ANOVA, followed by a multiple comparison test (Newman-Keuls).

\section{Results}

\section{Study population}

This study included samples from seventeen patients with ACC (main clinical characteristics reported in Table 1). Only two of the included ACC patients were children in which the presence of a genetic cause was not known (case 6 and 8; 9.5 and 4.2 years old respectively).

To describe the IGF pathway, the protein expression levels of IGF2, IGF1R and IGF2R were evaluated by IHC in the ACC samples. In twelve of these samples, the mRNA expression levels of $I G F 1, I G F 2, I G F 1 R, I R A, I R B, I G F 2 R$, $\operatorname{IGFBP} 1,2,3$ and 6 were evaluated by RT-qPCR.

\section{mRNA expression of the components of the IGF pathway in human ACC and NA samples}

The mRNA expression of several components of the IGF pathway was evaluated by RT-qPCR in 12 ACC samples and in 6 NA samples. As shown in Fig. 1, the expression levels of most of these IGF pathway components are quite variable in the different samples evaluated, although a high relative expression of $I G F 2$ was found in the majority of samples observed (mean 66,8 $\pm 106,4$; median levels 24.82; range 0.01-289.68). As compared with other receptors evaluated, the receptor expressed at highest levels within tumors was IRA in 7 of 12 samples (58.3\%), IGF2R in 3 (25\%), IGFIR in one (8.3\%) and IRB in the remaining one (8.3\%). Considering only the tyrosine-kinase receptors, IRA was the receptor expressed at highest levels in the majority

Table 1 IGF2, IGF1R and IGF2R protein expression in 17 adrenocortical cancer samples

\begin{tabular}{|c|c|c|c|c|c|c|c|c|c|}
\hline \multirow{2}{*}{$\begin{array}{l}\text { Patient } \\
\text { number }\end{array}$} & \multirow[t]{2}{*}{ Sex } & \multirow[t]{2}{*}{ Weiss } & \multirow{2}{*}{$\begin{array}{l}\text { Hormonal } \\
\text { secretion }\end{array}$} & \multicolumn{2}{|c|}{ IGF2 protein expression } & \multicolumn{2}{|c|}{ IGF1R protein expression } & \multicolumn{2}{|c|}{ IGF2R protein expression } \\
\hline & & & & Score & $\begin{array}{l}\text { Considerable } \\
\text { expression }\end{array}$ & Score & $\begin{array}{l}\text { Considerable } \\
\text { expression }\end{array}$ & Score & $\begin{array}{l}\text { Considerable } \\
\text { expression }\end{array}$ \\
\hline 1 & $\mathrm{~F}$ & 3 & $\mathrm{C}$ & 4 & Yes & 5 & Yes & 4 & Yes \\
\hline 2 & $\mathrm{~F}$ & 5 & $\mathrm{C}$ and $\mathrm{A}$ & 6 & Yes & 4 & Yes & 5 & Yes \\
\hline 3 & $\mathrm{~F}$ & 9 & A & 6 & Yes & 3 & No & 4 & Yes \\
\hline 4 & $\mathrm{M}$ & 6 & $\mathrm{C}$ and $\mathrm{A}$ & 3 & No & 6 & Yes & 5 & Yes \\
\hline 5 & $\mathrm{~F}$ & 6 & A & 5 & Yes & 5 & Yes & 5 & Yes \\
\hline 6 & $\mathrm{~F}$ & 7 & $\mathrm{C}$ and $\mathrm{A}$ & 6 & Yes & 6 & Yes & 5 & Yes \\
\hline 7 & M & 8 & none & 4 & Yes & 5 & Yes & 6 & Yes \\
\hline 8 & $\mathrm{~F}$ & 4 & A & 6 & Yes & 3 & No & 5 & Yes \\
\hline 9 & $\mathrm{~F}$ & 7 & none & 4 & Yes & 3 & No & 5 & Yes \\
\hline 10 & $\mathrm{~F}$ & 3 & none & 3 & No & 4 & yes & 5 & Yes \\
\hline 11 & $\mathrm{~F}$ & 7 & $\mathrm{C}$ and $\mathrm{A}$ & 6 & Yes & 4 & Yes & 5 & Yes \\
\hline 12 & $\mathrm{~F}$ & 5 & A & 5 & Yes & 6 & Yes & 6 & Yes \\
\hline 13 & $\mathrm{~F}$ & 7 & none & 4 & Yes & 3 & No & 4 & Yes \\
\hline 14 & $\mathrm{~F}$ & 8 & none & 6 & Yes & 3 & No & 5 & Yes \\
\hline 15 & M & 4 & A & 6 & Yes & 4 & Yes & 6 & Yes \\
\hline 16 & $\mathrm{~F}$ & 6 & none & 6 & Yes & 2 & No & 5 & Yes \\
\hline \multirow[t]{2}{*}{17} & M & 6 & $\mathrm{C}$ & 3 & No & 5 & Yes & 6 & Yes \\
\hline & & $\begin{array}{l}\text { Median 6; } \\
\text { Range 3-9 }\end{array}$ & & $\begin{array}{l}\text { Median 5; } \\
\text { Range 3-6 }\end{array}$ & $\begin{array}{l}\text { Frequency } 14 / 17 \\
(82 \%)\end{array}$ & $\begin{array}{l}\text { Median 4; } \\
\text { Range 2-6 }\end{array}$ & $\begin{array}{l}\text { Frequency } 11 / 17 \\
(65 \%)\end{array}$ & $\begin{array}{l}\text { Median 5; } \\
\text { Range 4-5 }\end{array}$ & $\begin{array}{l}\text { Frequency } 17 / 17 \\
(100 \%)\end{array}$ \\
\hline
\end{tabular}

$F$ female, $M$ male, $C$ cortisol, $A$ androgens 

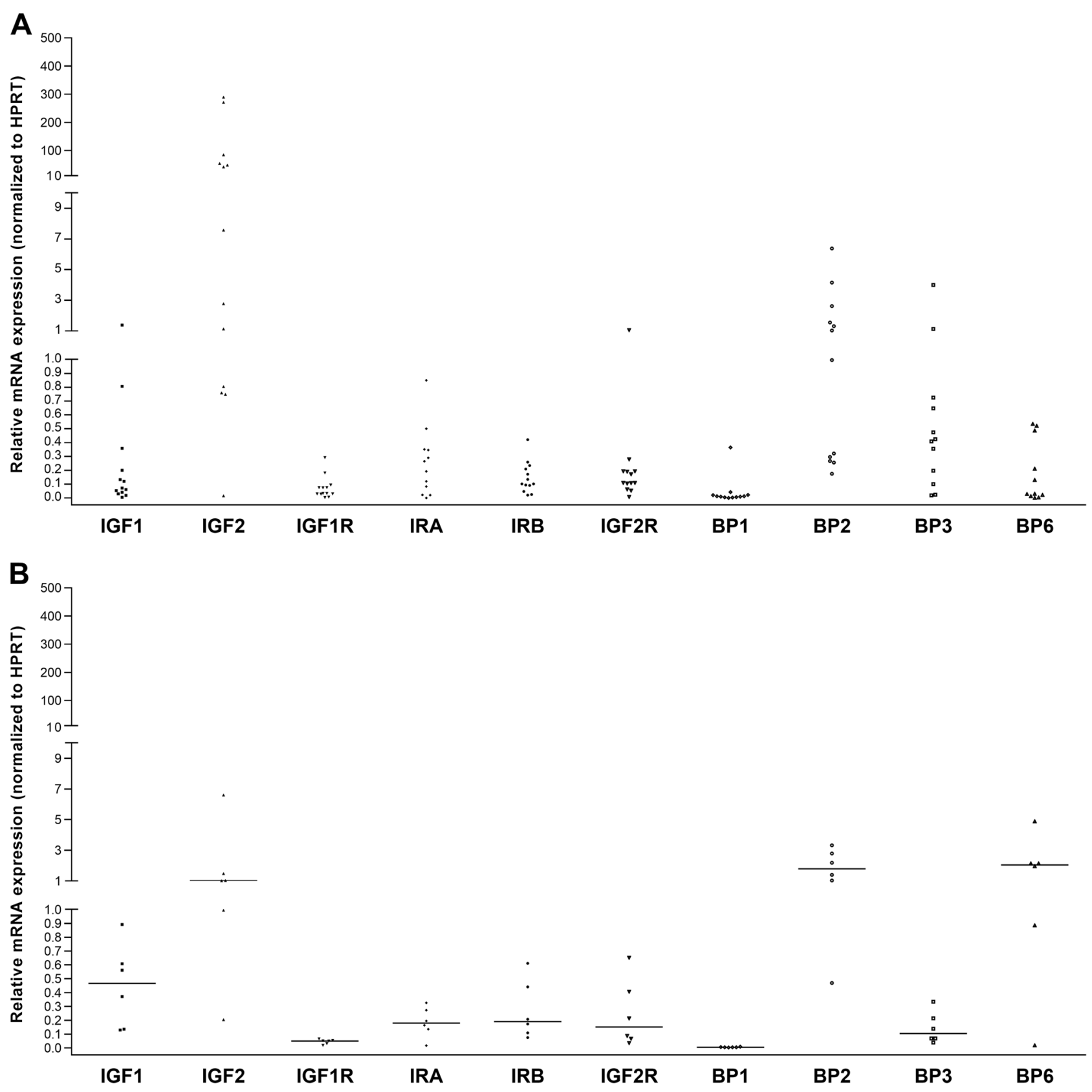

Fig. 1 mRNA expression levels of the main components of the IGFs and mTOR pathways (expressed as relative mRNA expression as normalized to the housekeeping gene HPRT) in a series of 17 human ACC samples $\mathbf{a}$ and in a series of 6 normal adrenals $\mathbf{b}$

of samples $(83 \%)$. Mean levels of IRA were significantly higher than mean levels of IGFIR $(0.25 \pm 0.26$ vs. $0.07 \pm$ $0.09 ; p<0.05)$. In all the evaluated samples, excepted for three cases, IRA/IRB ratio was higher than $1(2.19 \pm 1.59)$. As compared with other IGFBPs evaluated, the IGFBP expressed at highest levels within tumors was IGFBP2 in 7 of 12 samples (58.3\%); IGFBP3 in $4(33.3 \%)$ and IGFBP6 in only one case $(8.3 \%)$. Mean levels of IGFBP2 were significantly higher than the mean level of IGFBPI and IGFBP6 (1.16 \pm 1.9 versus $0.04 \pm 0.1 ; p<0.01$ and vs.
$0.18 \pm 0.07 ; p<0.05$, respectively). A negative correlation was found between IGF2 and IGFBP6 (rho: -0.8 ; $p<$ 0.003 ), whereas a positive correlation was found between $I G F I R$ and $I G F 2 R$ (rho: $0.7 ; p<0.009$ ); IGFIR and IRB (rho: $0.8 ; p<0.003$ ); IGFIR and IGFBPI (rho: $0.8 ; p<$ 0.001 ) and IRA and IGFBP2 (rho: $0.8 ; p<0.003$ ). No relationship was observed between the mRNA levels of the IGF components and any clinical parameter evaluated including hormone production, Weiss score, mitotic index and TNM. 
Fig. 2 Immunohistochemical detection of IGF2 a, IGF1R b and IGF2R $\mathbf{c}$ in a case of human adrenocortical carcinoma. $\mathbf{d}$ shows the absence of staining in the negative control. Magnification, $\times 200$
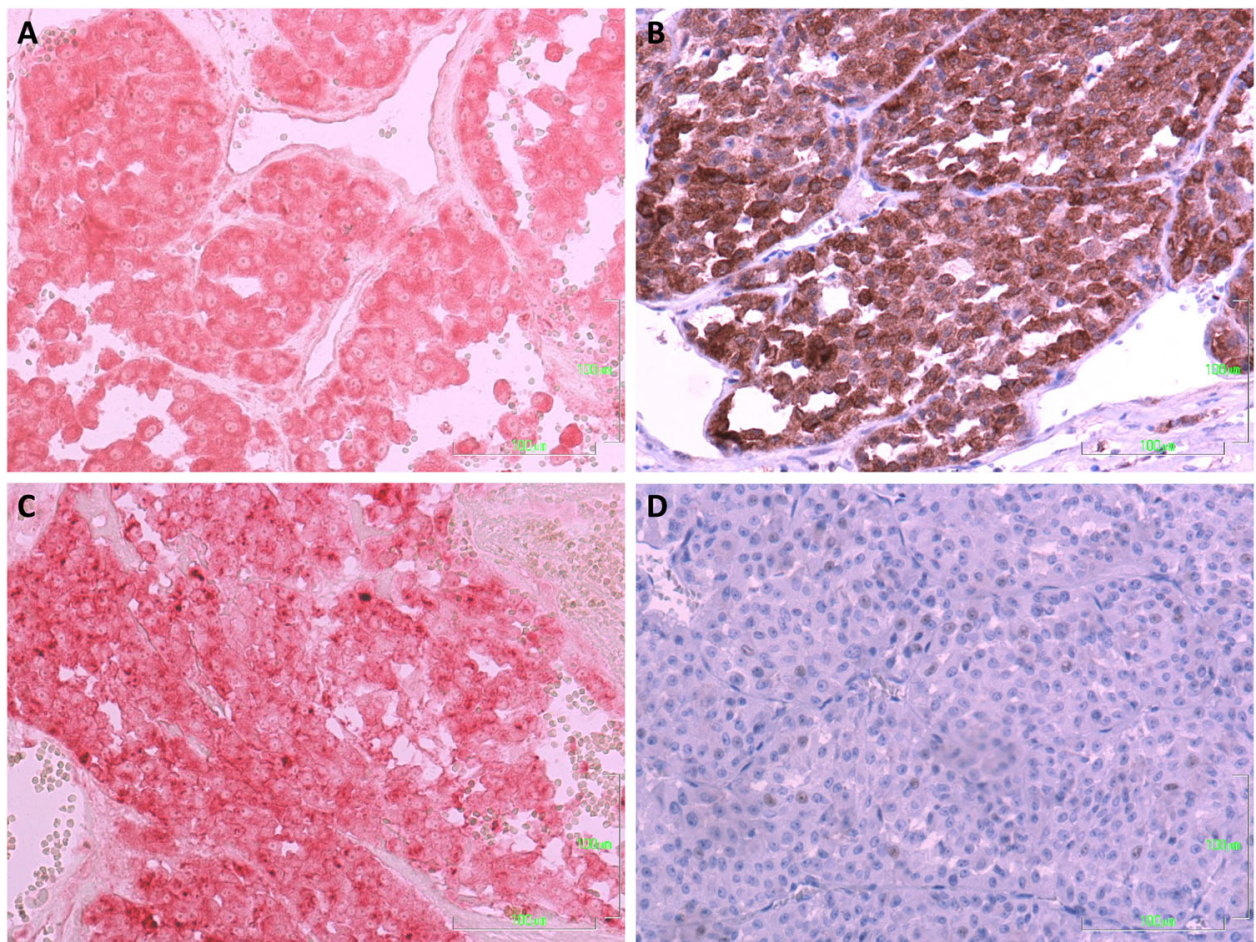

Mean levels of $I G F 1$ and IGFBP6 were significantly lower in ACCs as compared with NAs $(0.3 \pm 0.4$ versus $0.5 \pm 0.3 ; p<0.05$ and $0.2 \pm 0.2$ vs. $2.0 \pm 1.6, p<0.01$, respectively). Mean levels of $I G F 2$ were considerably higher in ACCs as compared with NAs $(66.8 \pm 103.6$ vs. $1.9 \pm 2.3 ; p<0.05)$, but this difference did not reach statistical significance, probably as a consequence of the small sample size and the high variation of $I G F 2$ levels within the tumor samples. Comparing the expression of the evaluated components of the IGF pathway in the 12 ACCs evaluated to the median value of each component in the NA, we observed an over expression of: IRA in 7 cases (58\%); IRB in $4(33,3 \%)$; IGF1 in $2(17 \%)$; IGF2 in 9 (75\%); IGFIR in $5(41.6 \%) ; I G F 2 R$ in $5(41.6 \%)$; IGFBPI in $10(83.3 \%)$; IGFBP2 in $3(25 \%)$; IGFBP3 in $9(75 \%)$ and IGFBP6 in none. In addition, 9 ACC samples showed an IRA-IRB ratio higher than the median value observed in normal adrenals.

\section{Protein expression of the components of the IGF pathway in human ACC samples}

The protein expression of IGF2, IGF1R and IGF2R was evaluated by IHC in 17 human ACCs. Table 1 summarizes the results of the IHC and the main clinical features of the evaluated patients. An intermediate to high staining for IGF2 (82\%; median score 5; range 3-6) and IGF1R (65\%; median score 4; range 2-6) was observed in most tumor tissues and for IGF2R (median score 5; range 4-6) in all ACCs. No correlations were observed between the expression of the different proteins that were evaluated and between these proteins and the main clinicalpathological characteristics of the corresponding patients. No correlations were observed between the protein and mRNA expression of IGF2, IGF1R and IGF2R although a trend to positive correlation was found between IGF2 protein and mRNA expression. Figure 2 shows an exemplary case of immunostaining in ACC. No particular expression has been observed in the two childhood ACC included in this series.

\section{Effects of dual IGF1R/IR inhibitor in human adrenocortical cell lines}

In both H295R and HAC15 cell lines linsitinib inhibited cell proliferation in a dose- and time-dependent manner (Fig. 3a, d). Linsitinib was slightly, but significantly, more potent in inhibiting cell proliferation in HAC15 compared to H295R. After 6 days of treatment in full medium the $\mathrm{IC}_{50}$ of linsitinib in $\mathrm{H} 295 \mathrm{R}$ was $1.5 \times 10^{-7} \mathrm{M}$ and in HAC15 $2.9 \times 10^{-8}$ M $(p<0.01)$. The maximal inhibition observed in H295R and HAC 15 was 90 and $95 \%$, respectively (not statistically significant; $p=0.3$ ). In both the H295R and HAC15 cells the potency of linsitinib and the maximal inhibition observed were similar in cell cultured in medium with high serum compared with cells cultured in medium with low serum (Fig. 3b, e). At the condition tested, linsitinib induced DNA-fragmentation in a dose-dependent manner in both H295R and HAC15 (Fig. 3c, f). 


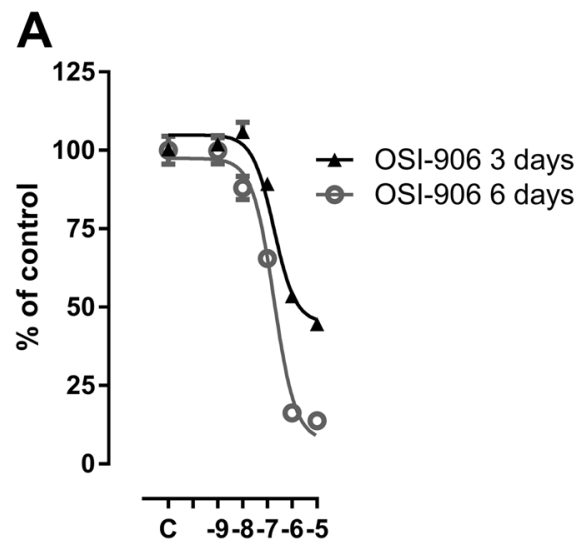

$\log [$ drug] (M)

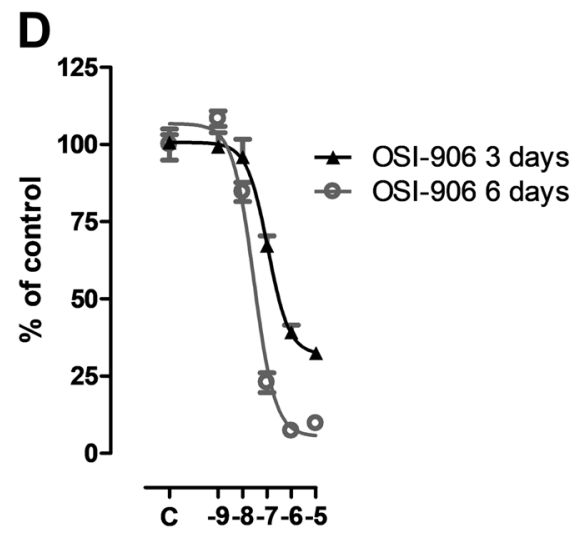

$\log$ [drug] (M)
B

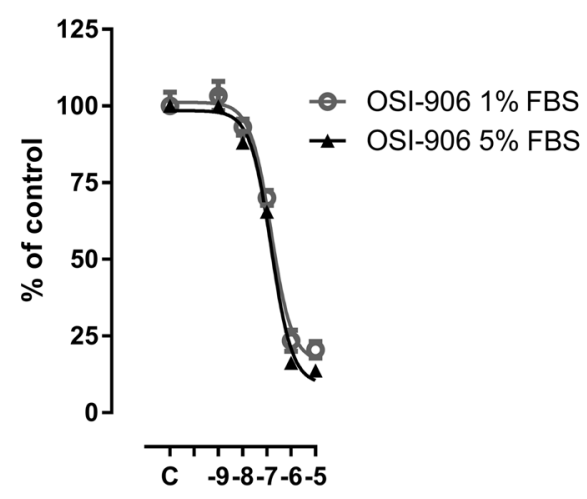

$\log [$ drug] (M)

E

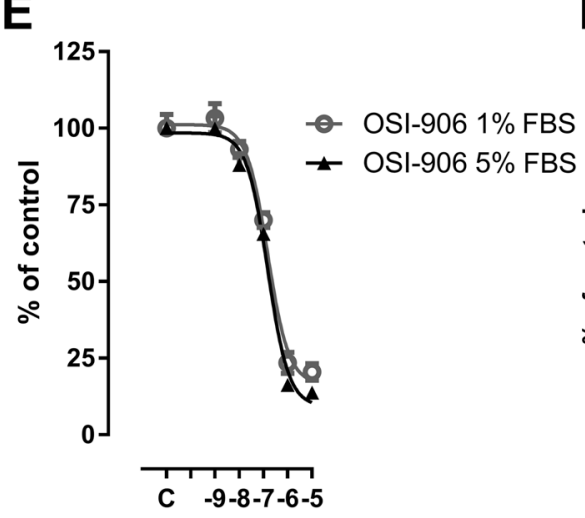

$\log$ [drug] (M)
C

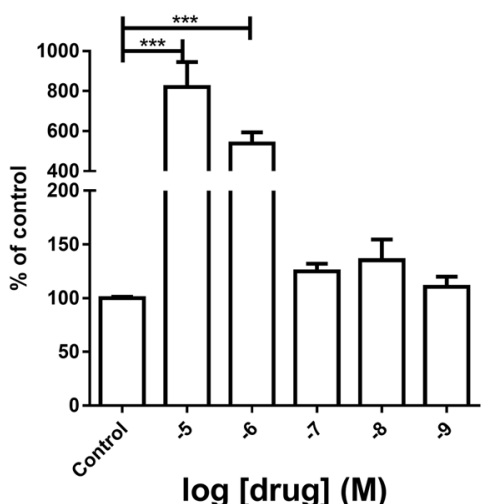

$\mathbf{F}$

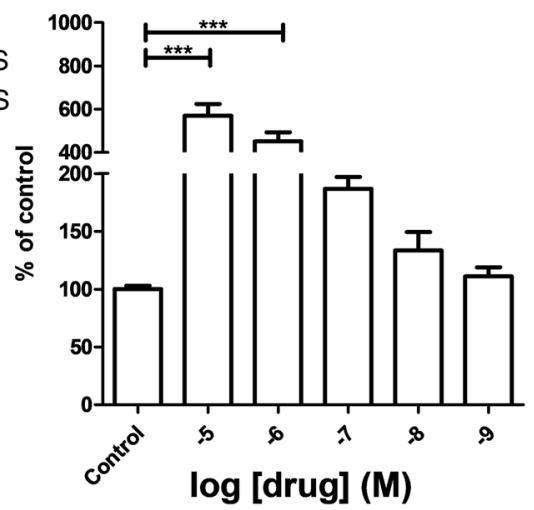

Fig. 3 Dose and time-dependent effect of linsitinib (OSI-906) treatment on H295R a, b and HAC15 d, e cell proliferation, expressed as DNA content/well after 3 days and 6 days a, $\mathbf{d}$ and after 6 days in medium with high or low serum $\mathbf{b}$, e. Dose-dependent effects of 3-day

\section{Effects of the dual IGF1R/IR inhibitor linsitinib in combination with mTOR inhibitors on human ACC cells}

Sirolumus and everolimus inhibited cell proliferation in H295R and HAC15 cells in a dose-dependent manner in both experimental conditions tested (high versus low serum concentration medium) data not shown. Sirolimus was slightly, but not significantly, more potent than everolimus. The potency of both compounds was similar in medium containing either high or low serum concentration. Selected doses of sirolimus or everolimus combined with linsitinib $5 \times 10^{-8} \mathrm{M}$ had statistically significant additive effect on cell proliferation (Fig. 4). Particularly both concentrations used of sirolimus and everolimus showed additive effect with linsitinib in inhibiting H295R and HAC cell proliferation when tested in medium containing low serum concentration (Fig. 4b, d, f, h). Only the highest concentrations used of mTOR inhibitors $\left(10^{-6} \mathrm{M}\right)$ showed some additive effect with linsitinib in inhibiting H295R and treatment with linsitinib on apoptosis of H295R $\mathbf{c}$ and HAC15 $\mathbf{f}$ cells, expressed as DNA fragmentation (normalized to the DNA content of each well). Data are expressed as the percentage of control and represent the mean \pm SEM. Control is set as $100 \%$. *** $p<0.001$

HAC cell proliferation when tested in medium containing high serum concentration (Fig. 4a, c, e, g).

At the condition tested, only the highest concentrations used of sirolimus $\left(10^{-6} \mathrm{M}\right)$ showed significant additive effect with linsitinib in increasing annexin $\mathrm{V}$, used as measure of apoptosis, in H295R (Fig. 5a). Everolimus did not show a statistically significant additive effect in increasing annexin $\mathrm{V}$ in H295R (Fig. 5b).

Linsitinib $\left(10^{-7} \mathrm{M}\right)$ alone or in combination with sirolimus $10^{-6} \mathrm{M}$ or $5 \times 10^{-9} \mathrm{M}$ significantly increased the proportion of cells in $\mathrm{G}_{0} / \mathrm{G}_{1}$ phase of the cell cycle $(p<$ $0.05, p<0.05$ and $p<0.01$ respectively). Linsitinib in combination with sirolimus $10^{-6} \mathrm{M}$ or $5 \times 10^{-9} \mathrm{M}$ significantly reduced the proportion of $\mathrm{G}_{2} / \mathrm{M}(p<0.05)$ (Fig. $5 \mathrm{c})$. Additionally the combined treatment with linsitinib and sirolimus showed a trend to have additive effects in inducing G1-cell cycle block. Statistically significant additive effects in increasing the proportion of cells in $\mathrm{G}_{0} / \mathrm{G}_{1}$ phase $(p<0.05)$ and reducing the proportion of cells in $\mathrm{G}_{2} / \mathrm{M}$ phase $(p<0.05)$ were observed when combining linsitinib 

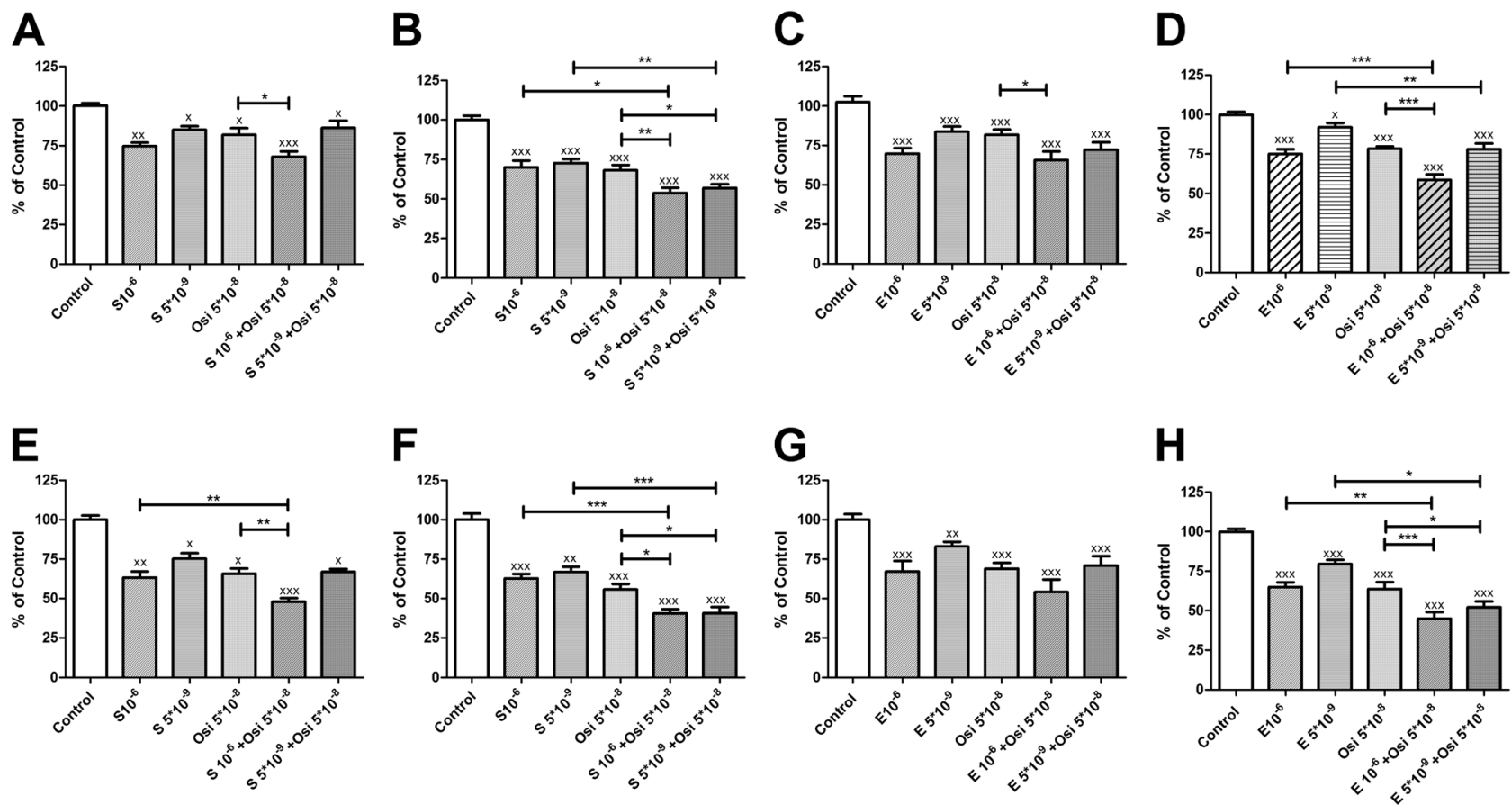

Fig. 4 Effect of linsitinib (OSI-906 indicated as Osi), alone or in combination with the mTOR inhibitors sirolimus (S) or everolimus (E), on H295R a-d and HAC15 e-h cell proliferation. Results are expressed as DNA content/well. Two different conditions have been

$10^{-7} \mathrm{M}$ and sirolimus $5 \times 10^{-9} \mathrm{M}$ as compared with sirolimus alone (Fig. 5c).

Linsitinib $\left(10^{-7} \mathrm{M}\right)$ alone or in combination with everolimus $10^{-6} \mathrm{M}$ or $5 \times 10^{-9} \mathrm{M}$ significantly increased the proportion of cells in $\mathrm{G}_{0} / \mathrm{G}_{1}$ phase of the cell cycle $(p<$ 0.01 ). Linsitinib alone or in combination with everolimus $10^{-6} \mathrm{M}$ or $5 \times 10^{-9} \mathrm{M}$ significantly reduced the proportion of cells in $\mathrm{G}_{2} / \mathrm{M}$ phase $(p<0.01)$ (Fig. 5d). Additionally, the combined treatment with linsitinib and everolimus showed significant additive effects in inducing G1-cell cycle block. Particularly, when combining linsitinib $10^{-7} \mathrm{M}$ and everolimus $10^{-6} \mathrm{M}$ or $5 \times 10^{-9} \mathrm{M}$, statistically significant additive effects in increasing the proportion of cells in $\mathrm{G}_{0} / \mathrm{G}_{1}$ phase ( $p<0.05$ and $p<0.01$, respectively) and in reducing the proportion of $\mathrm{G}_{2} / \mathrm{M}(p<0.05)$ as compared with everolimus alone were observed (Fig. 5d).

\section{Discussion}

The results of this study show that the majority of ACC express IGF2, IGF1R and IGF2R mRNA and protein and demonstrate IRA mRNA expression in these tumors, suggesting that factors such as IGF2R and IRA, not well described before, could interact with IGF2, potentially modulating the role of IGF2 in adrenocortical tumorigenesis. Mean levels of IGF1 and IGFBP6 were significantly

tested: medium with high $\mathbf{a}, \mathbf{c}, \mathbf{e}, \mathbf{g}$ or low serum $\mathbf{b}, \mathbf{d}, \mathbf{f}, \mathbf{h}$. The data are expressed as the percentage of control and represent the mean \pm SEM. Control is set as $100 \% .{ }^{*} p<0.05 ; * * p<0.01$; $* * * p<0.001 ;{ }^{\mathrm{x}} p<0.05$ vs. control; ${ }^{\mathrm{xx}} p<0.01$ vs. control; ${ }^{\mathrm{xxx}} p<0.001$ vs. control

lower in ACCs as compared with NAs. Additionally, this study demonstrates that treatment of human ACC cells with OSI-906, a dual IGF1R/IR inhibitor, reduces cell proliferation and that combined treatment with linsitinib and mTOR inhibitors can have additive antiproliferative effects.

A high mRNA and protein expression of IGF2 is found in most evaluated samples, in agreement with the already well-known IGF2 overexpression in 70-90\% of ACCs [817]. IGF1R protein expression was demonstrated in all the evaluated ACC samples and an intermediate-to-high staining was observed in more than $50 \%$ of cases. These data are in agreement with previous studies describing IGF1R expression in most ACCs $[11,14,38]$. The protein expression of IR in most ACCs has been previously described as well [38], however, to the best of our knowledge, the differential expression of IRA and IRB isoforms of the IR in ACCs, has never been explored. Unfortunately, to the best of our knowledge, there are currently no antibodies available to distinguish between the IR isoforms. We could therefore only evaluate IR isoform expression at mRNA level. While IRB is considered as the main mediator of metabolic effects of insulin and IGFs in adult tissue, IRA is an isoform of the IR, predominantly expressed during fetal development and is considered as an important mediator of growth-promoting effects of insulin and IGFs [19]. IRA has a higher affinity for IGF2 compared with the IGF1R and its expression in malignant tumor tissue has 

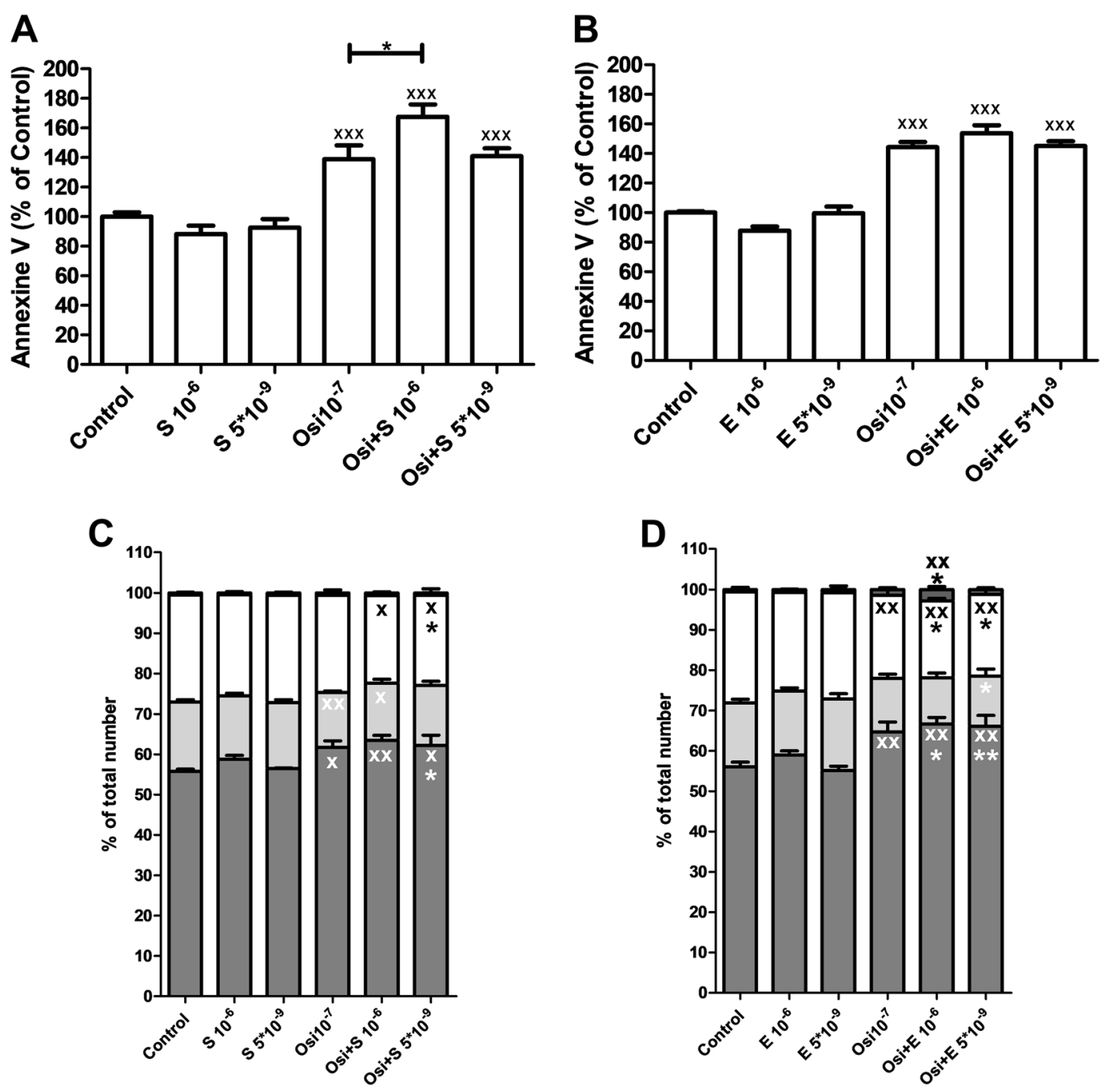

Fig. 5 a, b: Effect of linsitinib (OSI-906 indicated as Osi), alone or in combination with the mTOR inhibitors sirolimus $[(\mathrm{S})$; a $]$ or everolimus $[(\mathrm{E})$; b], on Annexin $\mathrm{V}$ as a measure of induction of apoptosis in H295R cell line. The data are expressed as the percentage of control and represent the mean \pm SEM. Control is set as $100 \%$. ${ }^{*} p<0.05$ vs.

been suggested to be involved in cancer development [19]. To our knowledge this is the first study demonstrating the presence of IRA in ACCs and showing that in these cancers, IRA is often expressed at higher level compared with IGF1R and IRB. The expression of IGF2 and the IGF1R has suggested a potential role of an IGF2-IGF1R autocrine loop in adrenocortical tumorigenesis [39]. The current study suggests a role of IRA as potential additional mediator of the IGF2 effects in ACC. However, in addition to the tyrosine kinase receptors involved in the IGF pathway, also IGF2R and IGFBPs could play a role in modulating the IGFs effects. The IGF2R serves a function in the degradation of IGF2, intracellular trafficking of lysosomal enzymes and activation of transforming growth factor beta. Down-

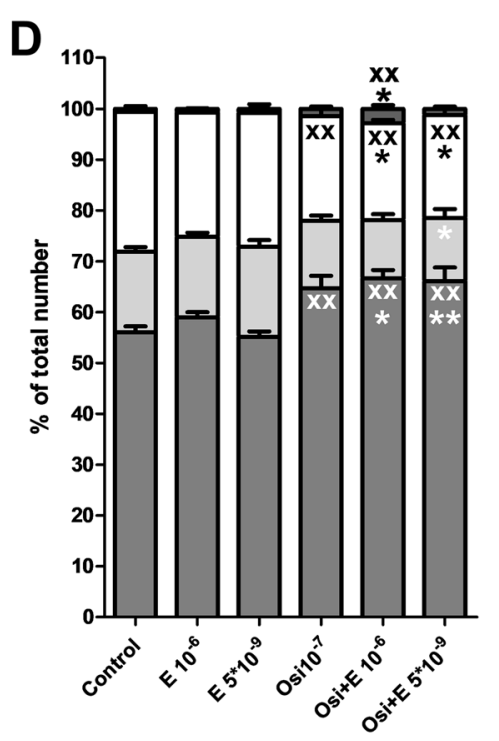

Osi alone; ${ }^{\mathrm{xxx}} p<0.001$ vs. control. c, $\mathbf{d}$ Effect of linsitinib (Osi), alone or in combination with the mTOR inhibitors sirolimus $\mathbf{c}$ or everolimus d, on cell cycle in H295R cell line. The data are expressed as mean \pm SEM. ${ }^{*} p<0.05$ and ${ }^{* *} p<0.01$ vs. S $\mathbf{c}$ or E $\mathbf{d}$ alone; ${ }^{\mathrm{x}} p<0.05$ and ${ }^{\mathrm{xx}} p$ $<0.01$ vs. control

regulation of IGF2R has been found in some type of cancers and it has been suggested that IGF2R could play a role as a tumor suppressor gene in some malignancies [40, 41]. Loss of heterozygosis at the locus of IGF2R gene has been reported to be a frequent event in ACC, supporting a potential role of IGF2R as a tumor suppressor gene also in ACC development [42]. However, a low protein expression of IGF2R in ACC has never been described. Conversely, the current study demonstrates the presence of a high IGF2R protein expression in most ACCs, suggesting that a high level of IGF2R protein might counteract the growthstimulating effects of IGF2 in adrenocortical tumorigenesis. In line with previously published data, in the current study a variable expression of IGFBPs was found in ACCs [10, 43]. 
Several correlations between the mRNA expression of different components of the IGF pathway were found suggesting the existence of common mechanisms of regulation. However no correlations with clinical-pathological parameters were found. This lack of correlation might be related to the small sample size and to the complexity of the IGF pathway. Among the IGFBPs evaluated, the IGFBP expressed at higher levels was IGFBP2, whereas IGFBP6 was expressed at lowest level. Additionally the expression of IGFBP6 was significantly lower in ACCs than in NAs. Therefore, whether high IGFBP2 and/or low IGFBP6 could play a role in the regulation of IGF pathway in adrenocortical tumorigenesis deserves further investigation. Additionally the IGFBP expressed at highest levels within ACC was IGFBP2, which has been recently suggested as a potential target for treatment in some type of cancers [44]. Therefore, in future studies it might be interesting to better explore the role of this and other IGFBPs as potential target for treatment in ACC as well.

The IGF pathway has been considered as one of the most promising targets for a novel medical treatment modality in patients with ACC [11, 14, 26, 45]. In preclinical models of ACC, two types of drugs targeting the IGF1R, i.e., NVPAEW541, a selective IGF1R kinase inhibitor and IMC-12, an IGF1R antibody, have been reported to have antiproliferative effects $[11,14]$, thus encouraging the development of clinical trials in ACC patients using drugs targeting the IGF pathway. The current study confirms that linsitinib (OSI-906), an IGF1R/IR inhibitor, inhibits the proliferation of the human ACC cell lines H295R and HAC15 in vitro already at a concentration lower than the concentrations reached in vivo in humans (about $5 \times 10^{-6}$ $\mathrm{M})$. However linsitinib has been recently tested in ACC patients in a phase III clinical trial (NCT00924989). The results of this study have been recently published [46] showing that only a very small subgroup of patients seems to benefit from treatment with this drug, and improvements in overall or progression-free survival were not observed. These apparent controversial results between preclinical and clinical studies, could be explained by several reasons. First, it might indicate that our preclinical models are not enough representative for the population of patients with ACC, because these tumors are heterogeneous. The role of the IGF pathway as a potential target for treatment in ACC might have been overestimated, as suggested by the fact that up-to-date in vivo experiments demonstrated that isolated IGF2 overexpression has no oncogenic potential [47]. Since disappointing results emerged in clinical trials adopting different types of drugs targeting the IGF pathway in different types of malignancies, despite apparently promising preclinical data [48], it could be hypothesized that current strategies adopted to target this pathway might still be inadequate. Indeed, biomarkers that can predict tumor response to IGF-targeting drugs, that might drive the selection of patient candidates to these drugs, have not been identified yet. Additionally, the complexity of the system could have been underestimated (such as the expression of potential regulators of the IGF pathway, as IGF2R in ACC) and the existence of interfering factors may not have been characterized yet. For example, in case of ACC patients the potential pharmacokinetic interactions between mitotane and drugs acting on the IGF pathway should be better investigated. Mitotane is a strong inducer of CYP3A4 and was shown to decrease bioavailability of sunitinib in patients with ACC [49]. Finally, targeting only the IGF pathway might not be sufficient to suppress cell growth because other pathways, that in part also interact with the IGF pathway (e.g. the mTOR pathway) are still activated. As such, before to finally declare a "game over" [47] for the role of IGF2 in adrenocortical tumorigenesis and as potential target for novel treatment in ACC patients, it could be probably useful to return to the bench and try to better explore the IGF pathway in its whole complexity. In line with this, the results of the current study point out that ACC express components of the IGF pathway, such as IRA and IGF2R, that have not been considered before.

In a previous study from our group, it was demonstrated that mTOR inhibitors inhibit cell proliferation in H295R and SW13 human ACC cell lines, but in H295R, probably as consequence of the IGF2 overexpression, this treatment could activate two potential pathways of escape to treatment with traditional mTOR inhibitors, i.e. the AKT and ERK pathways [25]. These data provide the rational for experiments combining mTOR inhibitors and drugs targeting the IGF pathway in ACC. In the current study the effects of linsitinib in combination with mTOR inhibitors were evaluated and the results of these experiments demonstrated that these compounds can have additive antiproliferative effects in some of the tested conditions. Particularly, additive antiproliferative effects were more pronounced when the experiments were performed using medium with low serum, suggesting that cell environment and the presence of growth factors different from IGF2 could influence the effects of these combination of compounds. These results are in line with a recently published phase I study demonstrating that a subgroup (about $40 \%$ ) of ACC patients treated with cixutumumab (IGF1R inhibitor) and temsirolimus experienced a long term disease stabilization [28]. These results suggest that treatment strategies combining mTOR inhibitors and linsitinib warrant further investigation, although considering the heterogeneous expression of the main components of the IGF pathway in the different ACC samples, the apparently modest antiproliferative effects observed at a low concentration of these compounds as well as the potential limits of the used human cell lines as model of human ACC, caution is recommended before to move from the bench to the bedside. 
A potential limitation of the current study is the small sample size. Although the number of samples included is reasonable considering the rarity of ACC, the reported results require confirmation in larger series of samples.

In conclusion, the present study describes the IGF pathway in ACC and explores the response to the combined treatment with the dual IGF1-/IR inhibitor linsitinib, and mTOR inhibitors in in-vitro models of ACC, demonstrating that human ACC express IGF2R and IRA which, in addition to IGF2 and its receptor IGF1R, might modulate the IGFs effects and linsitinib and mTOR inhibitors have additive antiproliferative effects.

Acknowledgements We thank D.M. Sprij-Mooij for the expert assistance with RT-qPCR. Research involving human participants and/or animals: This study was approved by the Medical Ethics Committee of the Erasmus MC

Author contributions Conception and design: MCDM; PMvK; RAF; LJH. Development of methodology: MCDM; PMvK; FD; DhoB; AMW; LJH. Acquisition of data: MCDM; PMvK; FD; DhoB; CP. Analysis and interpretation of data: MCDM; PMvK; RAF; WWdH; JAMJLJ; RP; AC; RRdK; DhoB; CP; LJH. Writing, review and/or revision of the manuscript: MCDM; RAF; WWdH; JAMJLJ; RP; AC; RRdK; DhoB; CP; LJH. Administrative, technical, or material support: MCDM; PMvK; FD; AMW. Study supervision: RAF; RP; LJH. All the authors approved the current manuscript as the final version to be published.

\section{Compliance with ethical standards}

Conflict of interest The authors declare that they have there is no conflict of interest.

Informed consent All patients gave written informed consent.

Publisher's note: Springer Nature remains neutral with regard to jurisdictional claims in published maps and institutional affiliations.

Open Access This article is distributed under the terms of the Creative Commons Attribution 4.0 International License (http://crea tivecommons.org/licenses/by/4.0/), which permits use, duplication, adaptation, distribution, and reproduction in any medium or format, as long as you give appropriate credit to the original author(s) and the source, provide a link to the Creative Commons license, and indicate if changes were made.

\section{References}

1. E. Baudin et al. Therapeutic management of advanced adrenocortical carcinoma: what do we know in 2011? Horm. Cancer 2 (6), 363-371 (2011)

2. K.Y. Bilimoria et al. Adrenocortical carcinoma in the United States: treatment utilization and prognostic factors. Cancer $\mathbf{1 1 3}$ (11), 3130-3136 (2008)

3. T. Else et al. Adrenocortical carcinoma. Endocr. Rev. 35(2), 282-326 (2014)

4. M. Fassnacht et al. European society of endocrinology clinical practice guidelines on the management of adrenocortical carcinoma in adults, in collaboration with the european network for the study of adrenal tumors. Eur. J. Endocrinol. 179(4), G1-G46 (2018)

5. A.M. Lerario, A. Moraitis, G.D. Hammer, Genetics and epigenetics of adrenocortical tumors. Mol. Cell. Endocrinol. 386(1-2), 67-84 (2014)

6. R. Weksberg, C. Shuman, J.B. Beckwith, Beckwith-Wiedemann syndrome. Eur. J. Hum. Genet. 18(1), 8-14 (2010)

7. T. Else, Association of adrenocortical carcinoma with familial cancer susceptibility syndromes. Mol. Cell. Endocrinol. 351(1), 66-70 (2012)

8. C. Gicquel et al. Structural and functional abnormalities at $11 \mathrm{p} 15$ are associated with the malignant phenotype in sporadic adrenocortical tumors: study on a series of 82 tumors. J. Clin. Endocrinol. Metab. 82(8), 2559-2565 (1997)

9. T.J. Giordano et al. Distinct transcriptional profiles of adrenocortical tumors uncovered by DNA microarray analysis. Am. J. Pathol. 162(2), 521-531 (2003)

10. N. Boulle et al. Increased levels of insulin-like growth factor II (IGF-II) and IGF-binding protein-2 are associated with malignancy in sporadic adrenocortical tumors. J. Clin. Endocrinol. Metab. 83(5), 1713-1720 (1998)

11. F.M. Barlaskar et al. Preclinical targeting of the type I insulin-like growth factor receptor in adrenocortical carcinoma. J. Clin. Endocrinol. Metab. 94(1), 204-212 (2009)

12. L.A. Erickson et al. Pathologic features and expression of insulinlike growth factor-2 in adrenocortical neoplasms. Endocr. Pathol. 12(4), 429-435 (2001)

13. A. Schmitt et al. IGFII and MIB1 immunohistochemistry is helpful for the differentiation of benign from malignant adrenocortical tumours. Histopathology 49(3), 298-307 (2006)

14. M.Q. Almeida et al. Expression of insulin-like growth factor-II and its receptor in pediatric and adult adrenocortical tumors. J. Clin. Endocrinol. Metab. 93(9), 3524-3531 (2008)

15. T.J. Giordano et al. Molecular classification and prognostication of adrenocortical tumors by transcriptome profiling. Clin. Cancer Res. 15(2), 668-676 (2009)

16. C. Laurell et al. Transcriptional profiling enables molecular classification of adrenocortical tumours. Eur. J. Endocrinol. 161(1), 141-152 (2009)

17. C. Gicquel et al. Rearrangements at the $11 \mathrm{p} 15$ locus and overexpression of insulin-like growth factor-II gene in sporadic adrenocortical tumors. J. Clin. Endocrinol. Metab. 78(6), 1444-1453 (1994)

18. R. Libe, J. Bertherat, Molecular genetics of adrenocortical tumours, from familial to sporadic diseases. Eur. J. Endocrinol. 153(4), 477-487 (2005)

19. A. Belfiore et al. Insulin receptor isoforms and insulin receptor/ insulin-like growth factor receptor hybrids in physiology and disease. Endocr. Rev. 30(6), 586-623 (2009)

20. B. Altieri, A. Colao, A. Faggiano, The role of insulin-like growth factor system in the adrenocortical tumors. Minerva Endocrinol. 44(1), 43-57 (2019)

21. M. Pollak, Insulin and insulin-like growth factor signalling in neoplasia. Nat. Rev. Cancer 8(12), 915-928 (2008)

22. G.V. Scagliotti, S. Novello, The role of the insulin-like growth factor signaling pathway in non-small cell lung cancer and other solid tumors. Cancer Treat. Rev. 38(4), 292-302 (2012)

23. P.F. Christopoulos, A. Corthay, M. Koutsilieris, Aiming for the Insulin-like Growth Factor-1 system in breast cancer therapeutics. Cancer Treat. Rev. 63, 79-95 (2018)

24. D.A. Guertin, D.M. Sabatini, Defining the role of mTOR in cancer. Cancer Cell. 12(1), 9-22 (2007)

25. M.C. De Martino, et al., The role of mTOR inhibitors in the inhibition of growth and cortisol secretion in human adrenocortical carcinoma cells. Endocr Relat Cancer 19(3), 351-364 (2012). 
26. M.C. De Martino et al. Role of the mTOR pathway in normal and tumoral adrenal cells. Neuroendocrinology 92(Suppl 1), 28-34 (2010)

27. M. Doghman et al. Regulation of insulin-like growth factormammalian target of rapamycin signaling by microRNA in childhood adrenocortical tumors. Cancer Res. 70(11), 4666-4675 (2010)

28. A. Naing et al. Insulin growth factor receptor (IGF-1R) antibody cixutumumab combined with the mTOR inhibitor temsirolimus in patients with metastatic adrenocortical carcinoma. Br. J. Cancer 108(4), 826-830 (2013)

29. M. Fassnacht et al. Limited prognostic value of the 2004 International Union Against Cancer staging classification for adrenocortical carcinoma: proposal for a Revised TNM Classification. Cancer 115(2), 243-250 (2009)

30. L.M. Weiss, Comparative histologic study of 43 metastasizing and nonmetastasizing adrenocortical tumors. Am. J. Surg. Pathol. 8(3), 163-169 (1984)

31. M. Fassnacht et al. Adrenocortical carcinoma: a clinician's update. Nat. Rev. Endocrinol. 7(6), 323-335 (2011)

32. R. Rasmussen. In: Rapid Cycle Real-Time PCR, Methods and Applications, ed. by S. Meuer, C. Wittwer, K. Nagakawara (Springer Press, Heidelberg, 2001), pp. 21-34

33. T.D. Schmittgen, K.J. Livak, Analyzing real-time PCR data by the comparative C(T) method. Nat. Protoc. 3(6), 1101-1108 (2008)

34. M.W. Pfaffl, A new mathematical model for relative quantification in real-time RT-PCR. Nucleic Acids Res. 29(9), e45 (2001)

35. M.C. De Martino et al. Characterization of the mTOR pathway in human normal adrenal and adrenocortical tumors. Endocr. Relat. Cancer 21(4), 601-613 (2014)

36. T. Wang, W.E. Rainey, Human adrenocortical carcinoma cell lines. Mol. Cell. Endocrinol. 351(1), 58-65 (2012)

37. L.J. Hofland, P.M. van Koetsveld, S.W. Lamberts, Percoll density gradient centrifugation of rat pituitary tumor cells: a study of functional heterogeneity within and between tumors with respect to growth rates, prolactin production and responsiveness to the somatostatin analog SMS 201-995. Eur. J. Cancer 26(1), 37-44 (1990)

38. T. Kamio et al. Immunoreactivity and receptor expression of insulinlike growth factor I and insulin in human adrenal tumors.
An immunohistochemical study of 94 cases. Am. J. Pathol. 138 (1), 83-91 (1991)

39. A. Logie et al. Autocrine role of IGF-II in proliferation of human adrenocortical carcinoma NCI H295R cell line. J. Mol. Endocrinol. 23(1), 23-32 (1999)

40. A.T. De Souza et al. M6P/IGF2R gene is mutated in human hepatocellular carcinomas with loss of heterozygosity. Nat. Genet. 11(4), 447-449 (1995)

41. W. Kiess et al. The insulin-like growth factor-II/mannose-6phosphate receptor: structure, function and differential expression. Adv. Exp. Med. Biol. 343, 175-188 (1993)

42. S. Leboulleux et al. Loss of heterozygosity at the mannose 6phosphate/insulin-like growth factor 2 receptor locus: a frequent but late event in adrenocortical tumorigenesis. Eur. J. Endocrinol. 144(2), 163-168 (2001)

43. V. Ilvesmaki et al. Expression of insulin-like growth factor binding protein 1-6 genes in adrenocortical tumors and pheochromocytomas. Horm. Metab. Res. 30(10), 619-623 (1998)

44. X. Yao et al. IGF-binding protein 2 is a candidate target of therapeutic potential in cancer. Tumour Biol. 37(2), 1451-1459 (2016)

45. T.C. Ribeiro, A.C. Latronico, Insulin-like growth factor system on adrenocortical tumorigenesis. Mol. Cell. Endocrinol. 351(1), 96-100 (2012)

46. M. Fassnacht et al. Linsitinib (OSI-906) versus placebo for patients with locally advanced or metastatic adrenocortical carcinoma: a double-blind, randomised, phase 3 study. Lancet Oncol. 16(4), 426-435 (2015)

47. C. Drelon, A. Berthon, P. Val, Adrenocortical cancer and IGF2: is the game over or our experimental models limited? J. Clin. Endocrinol. Metab. 98(2), 505-507 (2013)

48. B. Chmielowski, Insulin-like growth factor 1 receptor inhibitors: where do we come from? What are we? Where are we going? Cancer 120(16), 2384-2387 (2014)

49. V. Asp et al. Chiral effects in adrenocorticolytic action of o,p'DDD (mitotane) in human adrenal cells. Xenobiotica 40(3), 177-183 (2010) 